Revue d'histoire de l'Amérique française

RAS REVUE D.HISTOIRE DE L'AMÉRIQUE FRANÇAISE

\title{
Acte de mariage du patriote François-Marie Chevalier de Lorimier et d'Henriette Cadieux de Courville
}

\section{J.-J. Lefebvre}

Volume 22, numéro 1, juin 1968

URI : https://id.erudit.org/iderudit/302755ar

DOI : https://doi.org/10.7202/302755ar

Aller au sommaire du numéro

Éditeur(s)

Institut d'histoire de l'Amérique française

ISSN

0035-2357 (imprimé)

1492-1383 (numérique)

Découvrir la revue

Citer ce document

Lefebvre, J.-J. (1968). Acte de mariage du patriote François-Marie Chevalier de Lorimier et d'Henriette Cadieux de Courville. Revue d'histoire de l'Amérique française, 22(1), 93-93. https://doi.org/10.7202/302755ar d'utilisation que vous pouvez consulter en ligne. 


\section{ACTE DE MARIAGE DU PATRIOTE [FRANÇOIS-MARIE] CHEVALIER DE LORIMIER ET D'HENRIETTE CADIEUX DE COURVILLE *}

Le dix janvier mil huit cent trente deux, apres la publication d'un ban de mariage, sans empêchement ni opposition, la dispense de deux bans de Mariage ayant été obtenue de Messire Jacques Roque, grand Vicaire de l'évêque de Quebec, je soussigné Prêtre, autorise à cet effet, ayant pris le mutuel consentement par paroles de Présent de François Marie Thomas Chevaillier de Lorimier Ecuyer notaire de cette paroisse fils majeur de Guillaume Clément Edouard Verneuil de Lorimier, Ecuyer et de Dame Marie Marguerite Adelaïde Perrault domiciliés dans la paroisse de Saint Laurent d'une part;

Et de Demoiselle Marguerite Henriette Cadieux, fille mineure de feu Jean Marie Cadieux Ecuyer notaire et de Dame Marie Marguerite Institutrice de la susdite épouse de cette paroisse d'autre part, laquelle a donné son consentement audit Mariage, Les ai mariés suivant les lois et coutumes observés en la Sainte Eglise en presence de Guillaume Clement Edouard Verneuil de Lorimier écuyer père de l'épouse, de pierre Bibeau ecuyer avocat de Jean Baptiste Chamilly de Lorimier écuyer et de plusieurs autres soussignés avec les époux.

Henriette Cadieux de Courville

Guill. V. de Lorimier

P. Bibaud

P. Cadieux de Courville

L.G.C. de Lorimier

V. de Lorimier

George H. Cherrier

Flavie Bibaud

Odile Cherrier

Trefflé Cherrier

$$
\text { C. Fay, ptre }
$$

Chevalier de Lorimier

P. E. Leclere

Rachel Cadieux de Courville

Chamilly de Lorimier

Adelaïde de Lorimier

John B. Roy

F. M. chevalier de Lorimier

Emelie Roy

Marie Anne Roy

M ... de Lorimier

Romuald Cherrier

(Extr., Registres de la paroisse Notre-Dame de Montréal, 1832. Communication de J.-J. Lefebvre).

* Voir notre Revue, V : 115-117. 